\title{
Gastroenterologie up2date
}

\section{$3 \cdot 2020$}

\section{Leber / Galle / Pankreas 1}

\section{Diagnostik und Therapie des hepatozellulären Karzinoms}

Nada Abedin Jörg Trojan 


\section{Unter dieser Rubrik sind bereits erschienen:}

Virushepatitis: Chronische Hepatitis-B- und -D-Infektion

M. Demir, F. Tacke Heft 2/2020

Morbus Wilson und Hämochromatose S. Domschke, I. Mohr, U. Merle Heft 2/2020

Hepatitis-A- und -E-Virus-Infektionen - Altes und Neues

J. Hammig, H. Wedemeyer Heft 2/2020

Der unklare Leberrundherd M. Schultheiß, B. Bengsch, R. Thimme Heft 4/2019

Gallensteinerkrankungen F. Lammert, C. Gutt Heft 3/2019

Aktuelle Diagnostik und Therapiekonzepte für das Pankreaskarzinom A. Kestler, T. Seufferlein Heft 2/2019

Nicht alkoholische Fettlebererkrankung (NAFLD) - Ursachen, Diagnostik, Therapie M. Rau, A. Geier Heft 1/2019

Akutes Leberversagen M. Busch, K. Stahl, M. P. Manns

Heft 4/2018

Erhöhte Leberwerte A. Holstege Heft 3/2018

Renale und pulmonale Komplikationen der Leberzirrhose

R. Greinert, D. Greinert, A. Zipprich Heft 2/2018

Aktuelle Therapie der Hepatitis C: Wo stehen wir 2018?

K.-H. Peiffer, S. Zeuzem Heft 2/2018

Gallengangskarzinome M. M. Kirstein, A. Vogel Heft 4/2017

Autoimmunerkrankungen mit Leberbeteiligung M. Sebode, A. W. Lohse Heft 4/2017

IgG4-assoziierte Erkrankungen und Autoimmunpankreatitis J. Backhus, T. Seufferlein, A. Kleger Heft 3/2017

Transjugulärer intrahepatischer portosystemischer Shunt (TIPS) - Update 2016 I. Tesdal Heft 4/2016
Interventionelle Verfahren bei metastasiertem kolorektalem Karzinom J. Omari, M. Seidensticker, J. Ricke Heft 3/2016

Vaskuläre Erkrankungen der Leber M. Praktiknjo, J. Trebicka Heft 2/2016

Gutartige Lebertumoren M.-A Wörns, R. Kloeckner,

J. Marquardt Heft 1/2016

Leber und Gerinnung O. Mohamed, M. Dollinger, T. Seufferlein, M. Griesshammer Heft 1/2016

Chronische Pankreatitis - Update 2015 V. Keim Heft 4/2015

Gallensteinerkrankungen - Update 2015 F. Lammert,

M. Neubrand Heft 4/2015

Hepatorenales Syndrom und zirrhotische Kardiomyopathie D. Benten, V. Fuhrmann Heft 2/2015

Hepatozelluläres Karzinom - Update T. Greten Heft 1/2015

Hepatopulmonales Syndrom und portopulmonale Hypertension V. Fuhrmann, D. Benten Heft 1/2015

Interventionelle Therapie und stereotaktische Strahlentherapie von Lebermetastasen C. Wybranski, S. Lettmaier, J. Ricke Heft 4/2014

Hepatitis E P. Behrendt, H. Wedemeyer, M. Manns Heft 3/2014

Therapien bei Hepatitis C M. Schneider, C. Sarrazin Heft 2/2014

Akute Pankreatitis S. Teising, P. Layer, J. Keller Heft 4/2013

Adjuvante und palliative Therapie des Pankreaskarzinoms Update V. Kächele, T. Seufferlein Heft 3/2013

Medikamentös induzierte Leberschädigung $\mathrm{H}$. Spangenberg Heft 3/2013

\section{ALLES ONLINE LESEN}

Mit der eRef lesen Sie Ihre Zeitschrift: online wie offline, am PC und mobil, alle bereits erschienenen Artikel. Für Abonnenten kostenlos! https://eref.thieme.de/gastro-u2d

\section{IHR ONLINE-SAMMELORDNER}

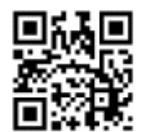

Sie möchten jederzeit und überall auf Ihr up2date-Archiv zugreifen? Kein Problem!

Ihren immer aktuellen Online-

Sammelordner finden Sie unter:

https://eref.thieme.de/F8661

\section{JETZT FREISCHALTEN}

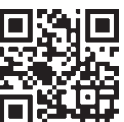

Sie haben Ihre Zeitschrift noch nicht freigeschaltet? Ein Klick genügt:

www.thieme.de/eref-registrierung 


\section{Diagnostik und Therapie des hepatozellulären Karzinoms}

Nada Abedin, Jörg Trojan

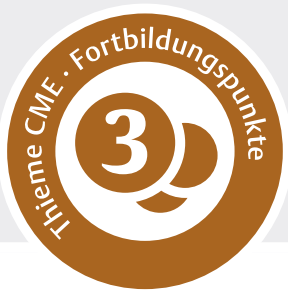

Primäre Tumorerkrankungen der Leber nehmen kontinuierlich zu, die häufigste Form ist das hepatozelluläre Karzinom. Überwiegend betroffen sind Männer mit einer viral, metabolisch und/oder durch Alkohol bedingten Leberzirrhose. In den letzten Jahren haben sich stadienabhängig viele verschiedene Therapiemodalitäten etabliert, die im Rahmen einer interdisziplinären Betreuung eingesetzt werden können. Dieser Beitrag gibt einen aktuellen Überblick.

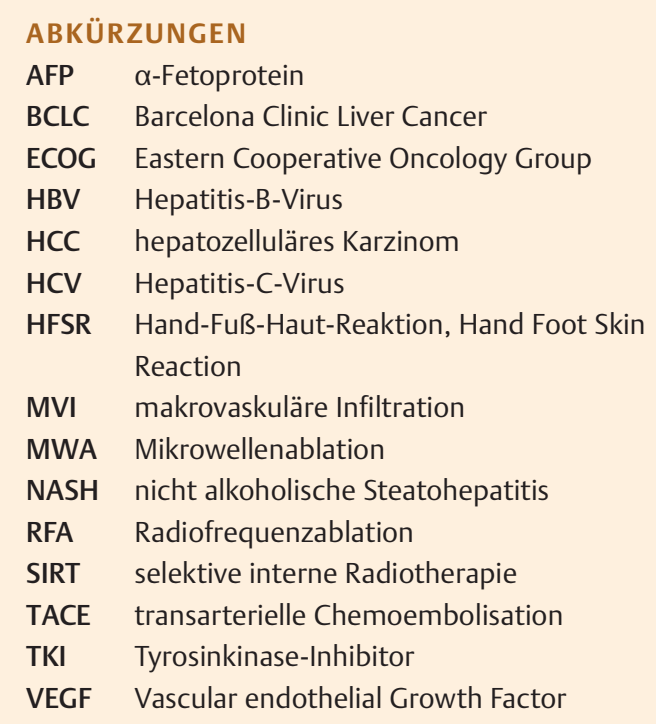

\section{Ursachen}

Das hepatozelluläre Karzinom (HCC) ist der häufigste primäre maligne Lebertumor mit einer zunehmenden Inzidenz. In 80-90\% der Fälle entwickelt sich ein HCC auf dem Boden einer Leberzirrhose. Ursachen hierfür sind meist eine chronische Hepatitis-C-Virus-Infektion oder Alkohol. Zunehmend gewinnen aber auch diätetische und Life-Style-Faktoren an Bedeutung, die maßgeblich zum Anstieg des Fettleber-assoziierten HCC beitragen [1].

\section{Pathogenese und Risikofaktoren}

Die Inzidenz des HCC ist in den letzten Jahren deutlich angestiegen. Allein in Deutschland wurde aktuell bei knapp 9000 Patienten die Erstdiagnose eines HCC gestellt, etwa 8650 HCC-assoziierte Todesfälle sind beschrieben. Weltweit erkrankten nach Angaben der WHO mehr als 840000 Patienten an einem HCC. Männer sind etwa 3bis 4-mal häufiger von einem HCC betroffen als Frauen, wobei der Anteil der erkrankten Frauen aktuell zunimmt. Das mittlere Erkrankungsalter liegt bei 60-65 Jahren [2].

\footnotetext{
Merke

Meist entwickelt sich ein HCC als Komplikation einer vorbestehenden Leberschädigung, in $80-90 \%$ der Fälle liegt eine Leberzirrhose zugrunde.
}

Seltener kann ein HCC auch ohne vorbestehende Lebererkrankung auftreten.

Die häufigsten Ursachen für eine Leberzirrhose sind eine chronische Infektion mit dem Hepatitis-C-Virus (HCV), eine chronische Infektion mit dem Hepatitis-B-Virus (HBV) und Alkohol [1]. Zunehmend gewinnt auch die nicht alkoholische Steatohepatitis (NASH) als Ursache für eine Leberzirrhose und für das HCC an Bedeutung. In den letzten Jahren ist die Zahl der HCC-Patienten mit vorbestehender metabolischer Lebererkrankung, insbesondere der NASH, beispielsweise als Folge eines Diabetes oder Adipositas, deutlich gestiegen. Mit Zunahme der zugrunde liegenden Erkrankungen wird für die nächsten Jahre ein weiterer Anstieg der HCC-Neuerkrankungen auf dem Boden der NASH prognostiziert [1,3]. Darüber hinaus gibt es seltenere Ursachen, wie z. B. eine Hämochromatose, Autoimmunerkrankungen oder andere chronische Lebererkrankungen. 
FALLBEISPIEL

Eine schlanke 62-jährige, gebürtig aus Ostasien stammende Patientin wird zur weiteren Abklärung einer unklaren Leberraumforderung vorgestellt. Wenige Wochen zuvor klagte die Patientin über plötzlich einsetzende Übelkeit, Erbrechen und starke Schmerzen im Oberbauch begleitet von einem Ikterus. Unter einer symptomatischen Therapie mit Paracetamol besserten sich die Beschwerden rasch. Bei Verdacht auf Abgang eines Gallenkonkrementes erfolgten extern zunächst eine Sonografie und eine MRT. Hier zeigten sich eine tumorverdächtige zentrale Leberveränderung sowie weitere Leberherde.

\section{Prävention}

Geeignete Hygienemaßnahmen und konsequente HBVImpfungen im Kindesalter können präventiv zur Verminderung der HCC-Inzidenz beitragen. Virale Infektionen sollten immer behandelt werden. Patienten mit einer HBV-Infektion sollten z. B. mit einem Nukleos(t)id-Analogon antiviral therapiert werden [1].

\section{Merke}

Sofern eine chronische HBV-Infektion vorliegt, sollte auch nach Entwicklung eines HCC eine dauerhafte antivirale Therapie durchgeführt werden, um die Leberfunktion zu erhalten.

Patienten mit einer chronischen HCV-Infektion können durch eine antivirale Therapie - derzeit als Standardtherapie Sofosbuvir und ein NS5A-Inhibitor - geheilt werden, wodurch das HCC-Risiko drastisch gesenkt werden kann. Patienten, die bereits eine Leberzirrhose entwickelt haben, weisen jedoch trotz Viruselimination weiterhin ein erhöhtes HCC-Risiko auf [4].

Die Therapie der metabolischen Lebererkrankung ist weiterhin von einer Lebensstiländerung geprägt. Diese besteht aus Bewegungssteigerung, Gewichtsreduktion und diätetischen Maßnahmen. Eine medikamentöse Therapie ist noch nicht etabliert. Bei Patienten mit nicht insulinabhängigem Diabetes hat Metformin möglicherweise einen HCC-protektiven Effekt [5].

Durch eine regelmäßige Aderlassbehandlung können das Zirrhose- sowie das damit verbundene HCC-Risiko bei Patienten mit Hämochromatose deutlich gesenkt werden.
Unabhängig von der Ätiologie der chronischen Lebererkrankung verschlechtert Alkoholkonsum auch in geringen Mengen den Verlauf chronischer Lebererkrankungen und erhöht die HCC-Inzidenz. Somit sollte bei jeder Form der Lebererkrankung eine strikte Alkoholkarenz eingehalten werden.

\section{Früherkennung}

Patienten mit bekannter chronischer Lebererkrankung profitieren erheblich von einer regelmäßigen fachärztlichen Überwachung.

Merke

Patienten mit einer Leberzirrhose sollten halbjährlichen qualifizierten sonografischen Verlaufskontrollen zugeführt werden, um die Entwicklung eines HCC möglichst frühzeitig zu erkennen $[1,6]$.

Die Sonografie kann durch die Bestimmung des $\alpha$-Fetoproteins (AFP) im Serum ergänzt werden. Daraus resultiert eine verbesserte Sensitivität (45 vs. 63\%) [7]. Eine alleinige AFP-Bestimmung im Rahmen der Früherkennung ist aufgrund der zu geringen Sensitivität nicht sinnvoll. Neuere Biomarker wie die Kombination von AFP, AFP-L3 (Lektin-reaktives AFP) und PIVKA-II (protein induced by vitamin $\mathrm{K}$ absence or antagonist II) weisen eine höhere Sensitivität für die HCC-Früherkennung auf. Sie werden derzeit in Studien evaluiert [8].

Die Überwachung von Patienten mit chronischer Lebererkrankung ohne Zirrhose ist sicherlich wünschenswert, derzeit jedoch oftmals nicht praktikabel. Die nicht invasive Bestimmung des Fibrosegrades erlaubt eine bessere Risikostratifizierung und kann deshalb als Entscheidungskriterium für eine HCC-Früherkennung Anwendung finden [9].

\section{Diagnosestellung bei HCC-Verdacht}

Die Diagnose des HCC kann durch bildgebende Verfahren gestellt werden. Hierzu ist die Kenntnis der HCC-Charakteristika (Tendenz zur Hämorrhagie, Nekrotisierung und multizentrisches Wachstum) und des typischen Kontrastmittelverhaltens notwendig. Zur Diagnostik werden kontrastverstärkte triphasische (arterielle, portalvenöse und parenchymatöse Phase) Bildgebungen eingesetzt. Dazu gehören die CT, die MRT, aber auch die kontrastmittelverstärkte Sonografie (CEUS) [1].

Merke

Ein HCC-typisches Perfusionsverhalten besteht aus einer arteriellen Hypervaskularisation und einer fehlenden Kontrastierung in der parenchymatösen Phase („wash out“). Dies ist bei vorhandener Leberzirrhose beweisend für ein HCC. 


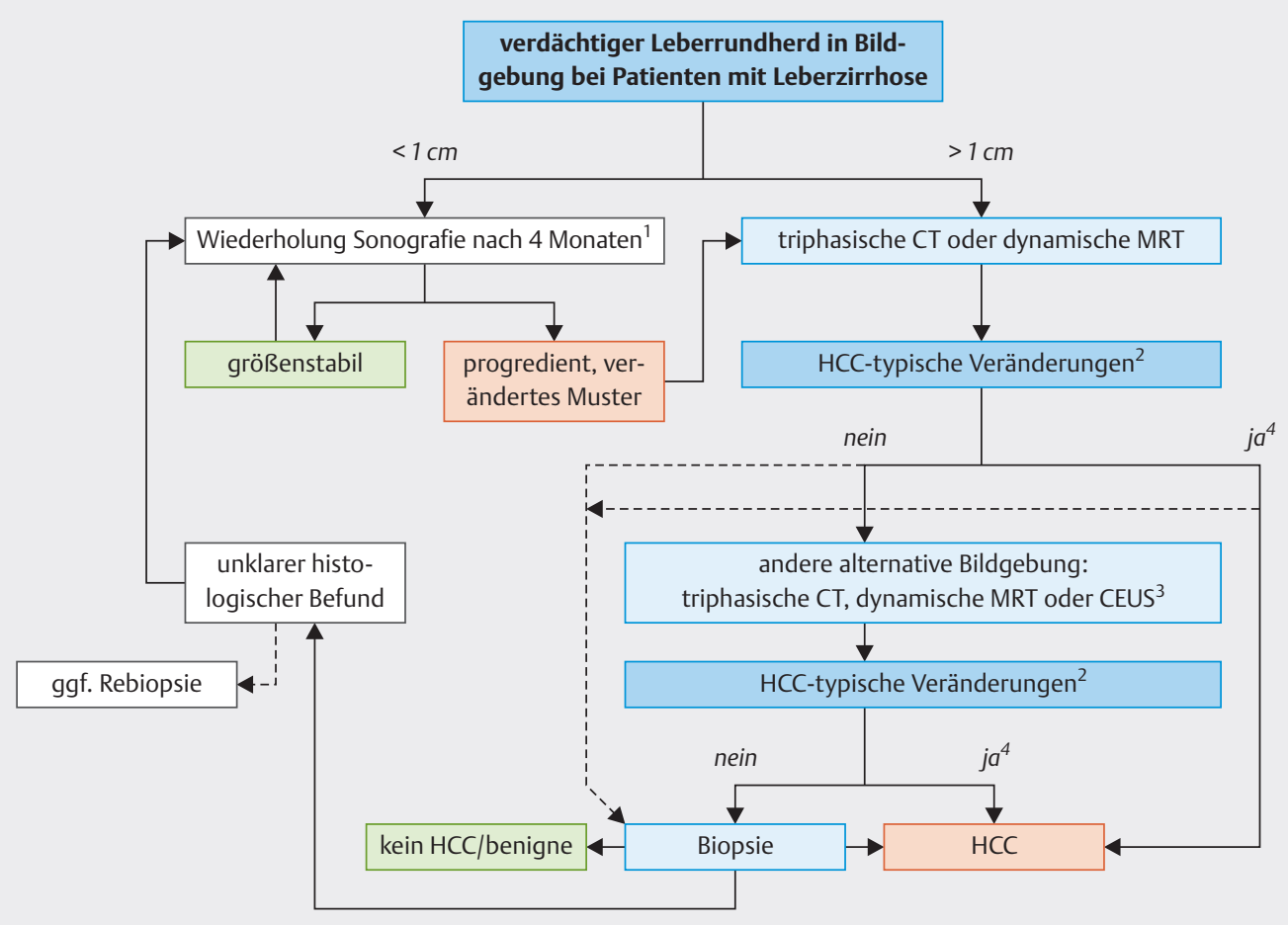

- Abb. 1 Diagnostischer Algorithmus bei Patienten mit Leberzirrhose bei Verdacht auf ein hepatozelluläres Karzinom (HCC) basierend auf der aktuellen EASL Guideline [1]. ${ }^{1}$ Für die Dauer eines Jahres, anschließend kann die Kontrolle bei stabilem Befund im Rahmen der halbjährlichen Sonografie erfolgen. ${ }^{2}$ HCC-typische Veränderungen: arterielle Hypervaskularisation sowie ein Kontrastmittelauswaschen („wash out“) in der portalvenösen Phase. ${ }^{3}$ CEUS: kontrastverstärkter Ultraschall. ${ }^{4}$ Optionale Biopsie, falls Standard am Zentrum. Quelle: Galle PR, Former A, Llovet J et al. EASL Clinical Practice Guidelines: Management of hepatocellular carcinoma. J Hepatol 2018; 69: 182-236.[rerif]

Die MRT erlaubt eine bessere Gewebecharakterisierung als die anderen Verfahren aufgrund des guten Weichteilkontrastes. In der nativen Untersuchung stellt sich hierbei ein suspekter Befund in den T2-gewichteten Sequenzen hyperintens und in den T1-gewichteten Sequenzen hypointens dar. Nach Gabe eines hepatozytenspezifischen Kontrastmittels spricht eine fehlende Kontrastmittelaufnahme in der biliären Phase für eine Entdifferenzierung des Tumors. Die PET/CT (Positronenemissionstomografie/Computertomografie) ist für die HCC-Diagnostik nicht sinnvoll.

\section{Diagnostischer Algorithmus}

Die individuelle Diagnostik bei Patienten mit einer Leberraumforderung und Leberzirrhose orientiert sich an den Empfehlungen der European Association for the Study of the Liver (EASL) ( $\triangleright$ Abb. 1) [1]:
- Unklare Leberherde $<1 \mathrm{~cm}$ sollten nach 4 Monaten kontrolliert werden.

- Bei Raumforderungen $\geq 1 \mathrm{~cm}$ in einer zirrhotischen Leber und eindeutigem Kontrastmittelverhalten im kontrastverstärkten MRT ist die Diagnose ohne histologische Sicherung zu stellen [1].

- Bei unklarem MRT-Befund wird eine weitere triphasische Bildgebung, z. B. CT, ggf. auch ergänzend CEUS, empfohlen.

- Leberherde, die $\geq 1 \mathrm{~cm}$ sind und untypische Perfusionsmerkmale zeigen, sollten bei vorliegender kurativer Behandlungsindikation in einer zweiten Bildgebung dargestellt werden.

- Liegt kein kurativer Ansatz vor, wird die Diagnose histologisch durch eine Biopsie bestätigt [1]. 


\section{FALLBEISPIEL}

\section{Diagnosestellung}

Unsere Patientin mit den tumorverdächtigen Leberherden war bis dato gesund, eine vorbestehende Lebererkrankung ist nicht bekannt. Labordiagnostisch zeigen sich eine leichte Transaminasenerhöhung (GOT 73 U/I, GPT 130 U/I) sowie eine Erhöhung der Cholestasewerte ( $\gamma$-GT 667 U/I, AP 321 U/I) bei minimal erhöhtem Bilirubinwert (1,13 mg/dl). Die Tumormarker CA 19-9 (81 U/ml) und AFP (46 ng/ml) sind erhöht. Bei positivem HBsAg ist eine niedrige Viruslast nachweisbar (HBV-DNA $290 \mathrm{IE} / \mathrm{ml}$ ).

Aufgrund der Verdachtsdiagnose eines intrahepatisch metastasierenden hilären Cholangiokarzinoms erfolgt eine ERCP (endoskopische retrograde Cholangiopankreatikografie). Bei dieser zeigt sich eine filiforme Stenose des Ductus hepaticus dexter, die durch Einlage einer Plastikprothese überbrückt wird ( $\bullet$ Abb. 2). Die darauffolgend durchgeführte sonografisch gesteuerte Feinnadelbiopsie erbringt die Diagnose eines mäßiggradig differenzierten hepatozellulären Karzinoms (immunhistochemisch CD34, Heppar1 und Glypican-3 positiv sowie CK7 negativ).

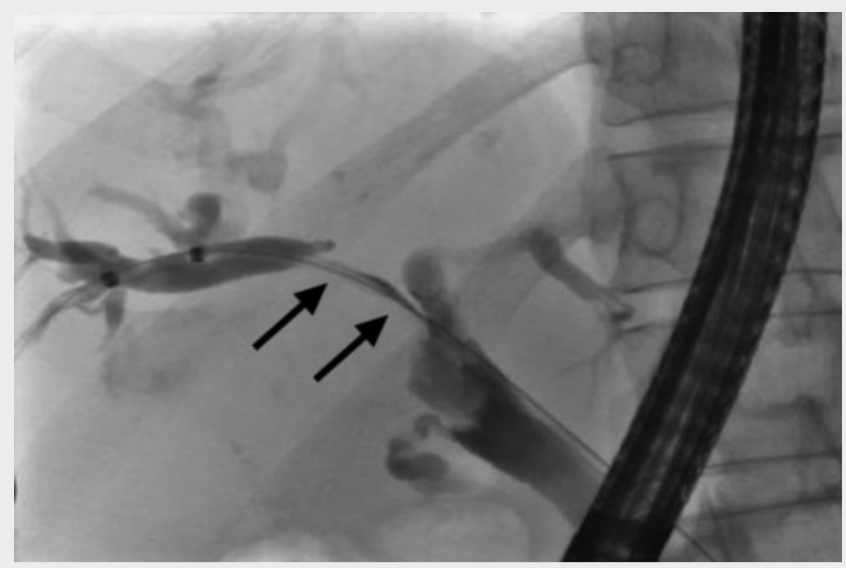

- Abb. 2 Darstellung einer filiformen Stenose des Ductus hepaticus dexter (Pfeile), die durch einen Plastikstent überbrückt wurde.

\section{Staging und Prognose}

Für das Staging und die intrahepatische Ausbreitungsdiagnostik des HCC wird eine dynamische MRT empfohlen. Diese ermöglicht die Einschätzung des Tumorausmaßes (solitärer Knoten vs. multiple Knoten) und eines möglichen Gefäßeinbruchs. Erfasst die MRT nicht das komplette Abdomen oder bestehen Kontraindikationen für eine MRT, sollte eine kontrastverstärkte AbdomenCT durchgeführt werden. Darüber hinaus wird ein kontrastverstärktes CT des Thorax zur Ausbreitungsdiagnostik empfohlen.

\section{Merke}

Für die Abschätzung der Prognose und der Therapieoptionen bei Patienten mit HCC sind das Tumorstadium und die Leberfunktion von Bedeutung.

Der aktuelle Therapiealgorithmus für Patienten mit HCC und Leberzirrhose richtet sich nach der BCLC-Einteilung (BCLC: Barcelona Clinic Liver Cancer), in die beide Faktoren einfließen $[1,6]$.

\section{Stadienabhängige Therapie}

Jeder Patient mit einem HCC soll in einer interdisziplinären Tumorkonferenz mit zwingender Beteiligung eines hepatobiliär erfahrenen Chirurgen, Gastroenterologen, Onkologen und Radiologen vorgestellt werden. Die Indikationsstellung zur Überweisung an ein Transplantationszentrum muss ebenfalls überprüft werden.

Bei der Therapie des HCC ist neben der Behandlung des Tumors insbesondere auch der Erhalt der häufig bereits bei Diagnosestellung eingeschränkten Leberfunktion von großer Bedeutung.

\section{Operative Therapien}

Resektion

Die Resektion gehört zu den potenziell kurativen Therapieoptionen und stellt insbesondere im Stadium BCLC 0 und BCLC A die bevorzugte Therapieoption dar. Sie sollte immer durch einen hepatobiliären Chirurgen eingeschätzt werden. Bei Patienten mit HCC ohne Leberzirrhose sollte man eine primäre Resektion auch bei großen Tumoren anstreben.

\section{Cave}

Bei Patienten mit vorbestehender Leberzirrhose ist eine Resektion nur bei erhaltener Leberfunktion möglich.

Darüber hinaus sind die allgemeine Operabilität und die Leberfunktionsreserve zu beachten [1]. Chirurgisch-anatomische Besonderheiten, wie die Infiltration aller 3 Lebervenen oder die Infiltration bzw. Miteinbeziehung der Hilusstrukturen, die einer R0-Resektion entgegenstehen, müssen vom hepatobiliären Chirurgen ebenfalls abgewogen werden. Sonderformen, die vor allem junge Patienten betreffen, wie das fibrolamelläre Karzinom, haben eine schlechte Prognose.

Patienten mit einem potenziell resektablen HCC in ungünstiger Lokalisation oder mit eingeschränkter Leberfunktion werden mit einer Thermoablation behandelt. 


\section{Lebertransplantation}

Für Patienten mit einer Leberzirrhose und einem HCC innerhalb der sog. Mailand-Kriterien kommt eine Lebertransplantation infrage. Die Mailand-Kriterien besagen: ein Herd $<5 \mathrm{~cm}$ oder maximal 3 Herde, jeder einzelne $<3 \mathrm{~cm}$; keine Fernmetastasierung oder Gefäßinfiltration. Auch Patienten mit mehr oder größeren Tumoren („Upto-seven“-Kriterien: Summe des Durchmessers des größten Herdes [in cm] und Anzahl der HCC-Herde $\leq 7$ ) profitieren von einer Transplantation [10]. In Deutschland ist aufgrund der rechtlichen Rahmenbedingungen (Richtlinien der Bundesärztekammer zur Lebertransplantation) jedoch nur eine Transplantation innerhalb der MailandKriterien möglich [11].

Die 3-Jahres-Überlebensraten nach Resektion und Transplantation liegen beide bei ca. $66 \%$. Nach 5 Jahren weisen Transplantatempfänger ein besseres Überleben im Vergleich zu resezierten Patienten auf (61 vs. 50\%) [10]. Die zunehmende Anzahl von Patienten mit HCC auf der Transplantationswarteliste und die limitierte Anzahl von Spenderorganen führen derzeit zu längeren Wartezeiten bis zur Transplantation. Um das Risiko für einen Tumorprogress zu reduzieren, erfolgt deshalb in der Regel eine TACE-Therapie (transarterielle Chemoembolisation) als Bridging bis zur Transplantation [1,6].

\section{Lokoregionäre Therapieverfahren}

\section{Thermoablation}

Bei Kontraindikationen gegen ein chirurgisches Verfahren und Vorliegen eines frühen HCC-Stadiums soll primär eine Thermoablation des Tumors durchgeführt werden. Diese erfolgt mittels Mikrowellen- (MWA) oder Radiofrequenzablation (RFA). Die Rezidivraten nach Ablation und Resektion unterscheiden sich nicht wesentlich voneinander. Eine Ablation kann bei bis zu 3 HCC-Herden mit einem maximalen Durchmesser von $3 \mathrm{~cm}$ durchgeführt werden.

\section{Chemoembolisation}

Bei Patienten mit einer Child-Pugh-A- oder Child-Pugh-BLeberzirrhose wird bei Herden $>3 \mathrm{~cm}$ und $<5 \mathrm{~cm}$ vor der Ablation eine Chemoembolisation empfohlen. Auch bei größeren Tumoren kann das Überleben durch eine zusätzliche Chemoembolisation verbessert werden [1].

Bei der selektiven Chemoembolisation erfolgt im Rahmen einer radiologischen Intervention die Kombination aus

- einer lokalen intraarteriellen Infusion von Chemotherapeutika (meist Doxorubicin oder Mitomycin C) und

- der Embolisation der vaskulären Versorgung („feeding vessel“) des Tumors durch Mikropartikel.
Neben der konventionellen Lipiodol-basierten transarteriellen Chemoembolisation (TACE) können im Rahmen der Intervention auch Drug-eluting Beads eingesetzt werden.

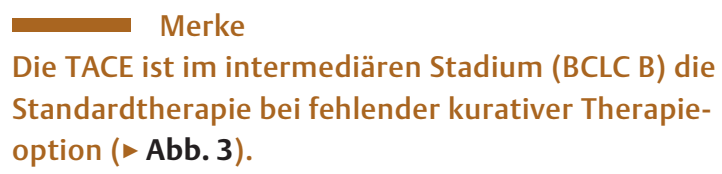

Vor Einleitung einer TACE sollten Kontraindikationen ausgeschlossen werden (s. „Praxis - TACE: Kontraindikationen“). Studien haben für die TACE im Vergleich zur supportiven Behandlung eine Verlängerung des medianen Überlebens von 16 auf 19 Monate gezeigt. In vielen retrospektiven Arbeiten an einem hochselektionierten Patientengut wurden sogar Überlebenszeiten von bis zu 4 Jahren beschrieben. In Einzelfällen kann eine TACE auch im fortgeschrittenen Stadium sinnvoll sein, eine solche Entscheidung soll jedoch interdisziplinär diskutiert werden. Bei fehlendem Ansprechen sollte die TACE nach spätestens 2 Therapiezyklen beendet werden, um eine weitere Beeinträchtigung der Leberfunktion zu vermeiden.

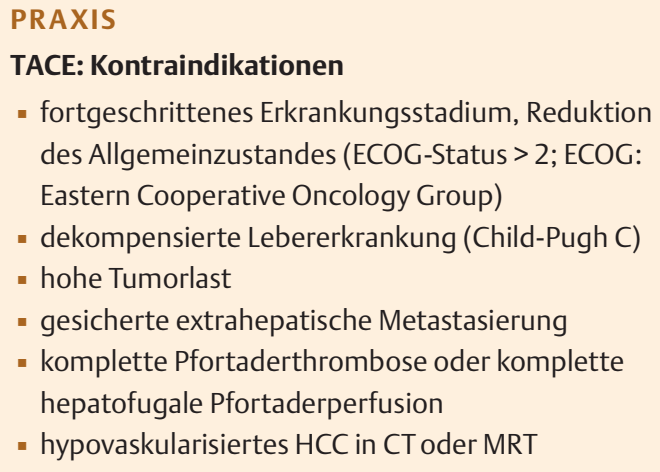

\section{Selektive interne Radiotherapie}

Die selektive interne Radiotherapie (SIRT; alternative Bezeichnung: transarterielle Radioembolisation, TARE) ist ein weiteres intraarterielles Therapieverfahren, das jedoch nur an wenigen Zentren verfügbar ist $[1,6]$. Hierbei erfolgt eine arterielle Applikation von radioaktiv (Yttrium-90) beladenen Sphären aus Glas oder Harz in die tumorversorgenden Gefäße. Ein Einsatz ist nur sinnvoll bei großen Tumoren im intermediären Stadium, ggf. auch bei Portaderthrombosen, nicht jedoch als „Salvage“-Therapie nach Progression unter TACE. Eine SIRT wird von den Patienten in der Regel gut vertragen [12]. 


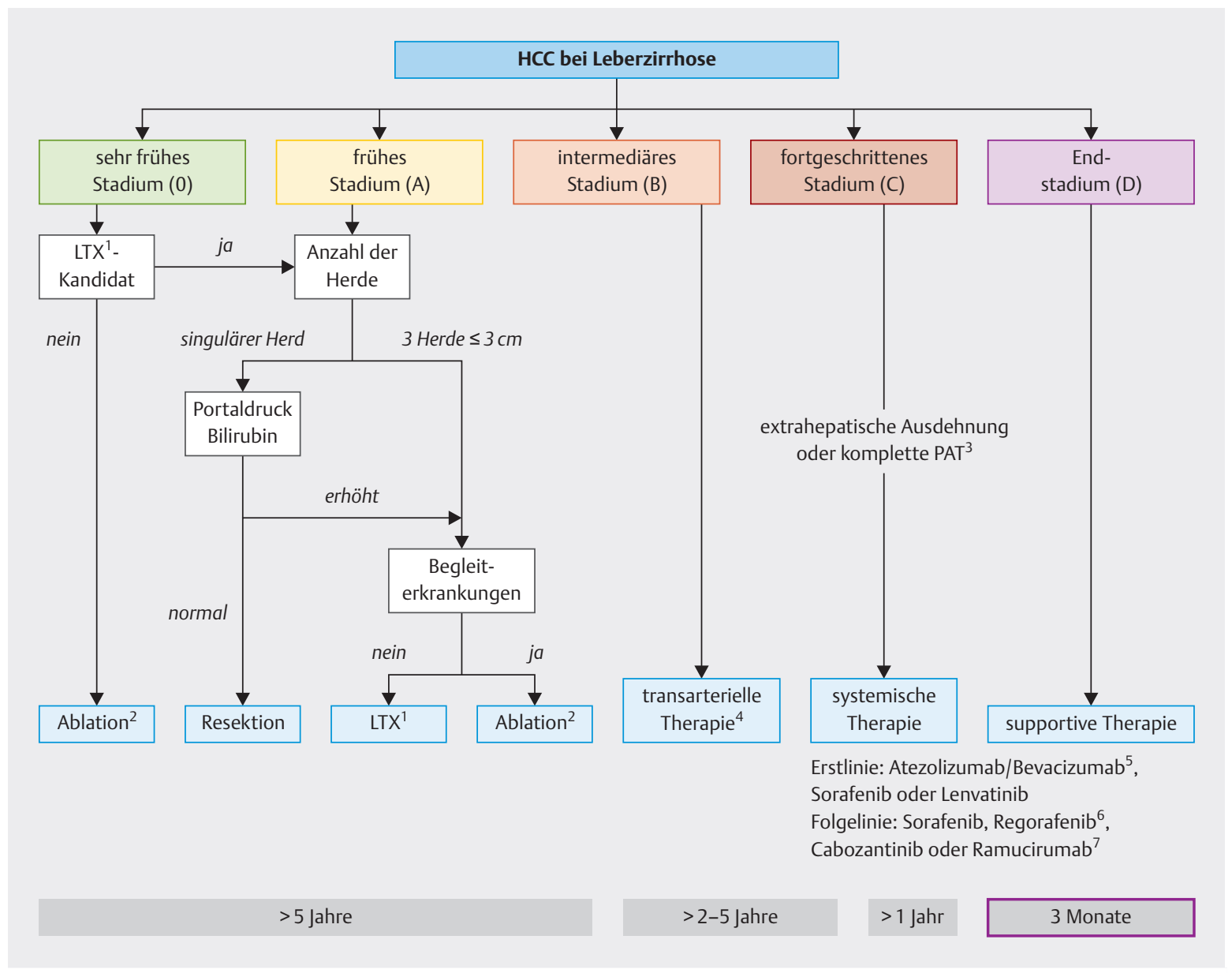

- Abb. 3 Therapiealgorithmus bei Patienten mit Leberzirrhose und hepatozellulärem Karzinom (HCC) in Anlehnung an die BCLCEinteilung (BCLC: Barcelona Clinic Liver Cancer). Daten nach [1]. ${ }^{1}$ LTX: Lebertransplantation. ${ }^{2}$ Mikrowellen- oder Radiofrequenzablation. ${ }^{3}$ PAT: Pfortaderthrombose. ${ }^{4}$ Transarterielle Chemoembolisation (Standard) oder in Einzelfällen selektive intraarterielle Radioembolisation. ${ }^{5}$ EMA-Zulassung im 3. Quartal 2020 erwartet. ${ }^{6}$ Bei Sorafenib-Verträglichkeit. ${ }^{7}$ Bei AFP $\geq 400$ ng/ml.

Merke

Aufgrund des hohen Rezidiv- bzw. Progressionsrisikos sind sowohl nach kurativ intendierter als auch nach transarterieller Therapie regelmäßige Verlaufskontrollen im 3- bis 6-Monats-Intervall mittels dynamischer MRT oder CT erforderlich [1].

\section{Systemische Therapien}

In den letzten 3 Jahren wurden große Fortschritte im Bereich der systemischen Therapie des HCC erzielt. Seit 2008 gab es für 10 Jahre mit Sorafenib nur eine mögliche Systemtherapie. Heute sind verschiedene Substanzen sowohl für die Erstlinientherapie als auch für Folgetherapielinien verfügbar. Neben Tyrosinkinase-Inhibitoren (TKI) und einem VEGFR2-Antikörper (VEGFR2: Vascular Endothelial Growth Factor Receptor 2) wird in Kürze auch eine Immuntherapie als Kombinationsbehandlung zur Verfügung stehen. Die Wahl der systemischen Therapie hängt deshalb von unterschiedlichen Faktoren ab ( $\triangleright$ Tab. 1).

\section{Erstlinientherapie}

Bei Patienten mit einem HCC im fortgeschrittenen Tumorstadium (BCLC C) war der VEGFR/BRAF-Tyrosinkinase-Inhibitor Sorafenib lange Jahre die Standard-Erstlinientherapie. Das Gesamtüberleben stieg im Vergleich zu Placebo von 7,9 auf 10,7 Monate [13]. Viele Behandler bevorzugen eine einschleichende Dosierung („step up“). Zur Anwendung bei Patienten mit mäßig eingeschränkter Leberfunktion (Child-Pugh B, 7 und 8 Punkte) liegen nur wenige Erfahrungen vor; der Nutzen in dieser Gruppe ist stark eingeschränkt $[1,6]$.

Als weiteres Medikament in der Erstlinientherapie wurde 2018 der Multi-Tyrosinkinase-Inhibitor Lenvatinib zugelassen [14]. Unter der Therapie mit Lenvatinib verdoppelte sich das mediane progressionsfreie Überleben im Vergleich zu Sorafenib von 3,7 auf 7,4 Monate. Darüber hinaus zeigte sich ein signifikanter Vorteil in der objektiven Ansprechrate, bei der Lenvatinib mit 24,1\% Sorafenib mit $9,2 \%$ deutlich überlegen war. 
\ab. 1 Erstlinien- und Zweitlinientherapien des hepatozellulären Karzinoms (HCC), Indikationen und Nebenwirkungen.

\begin{tabular}{|c|c|c|}
\hline Substanz und Art der Applikation & Indikation & häufige Nebenwirkungen \\
\hline \multicolumn{3}{|l|}{ Erstlinie } \\
\hline $\begin{array}{l}\text { Sorafenib } \\
\text { Tablette, } 400 \text { mg } 2 \text { × täglich }\end{array}$ & $\begin{array}{l}\text { - HCC im Stadium BCLCC } \\
\text { - Child-Pugh A } \\
\text { - ECOG }<2\end{array}$ & $\begin{array}{l}\text { - Hauttoxizität (HFSR) } \\
\text { - Diarrhö } \\
\text { - Hypertonus } \\
\text { - Appetitverlust } \\
\text { - Fatigue } \\
\text { - Alopezie }\end{array}$ \\
\hline $\begin{array}{l}\text { Lenvatinib } \\
\text { Tablette, } 8 \mathrm{mg}(\leq 60 \mathrm{~kg} \mathrm{KG}) \text { oder } \\
12 \mathrm{mg}(>60 \mathrm{~kg} \mathrm{KG}) \text { täglich }\end{array}$ & $\begin{array}{l}\text { - HCC im Stadium BCLCC } \\
\text { - Child-Pugh A } \\
\text { - ECOG } \leq 1 \\
\text { - keine Pfortaderhauptstamminvasion } \\
\text { - Tumorlast< }<50 \%\end{array}$ & $\begin{array}{l}\text { - Hypertonus } \\
\text { - Diarrhö } \\
\text { - Appetitverlust } \\
\text { - Gewichtsverlust } \\
\text { - Fatigue } \\
\text { - Hauttoxizität (v.a. HFSR) }\end{array}$ \\
\hline $\begin{array}{l}\text { Atezolizumab } 1200 \mathrm{mg}+ \\
\text { Bevacizumab } 15 \mathrm{mg} / \mathrm{kg} \mathrm{KG}^{1} \\
\text { Infusion, alle } 3 \text { Wochen }\end{array}$ & $\begin{array}{l}\text { - HCC im Stadium BCLCC } \\
\text { - Child-Pugh A } \\
\text { - ECOG 0-1 } \\
\text { - Ausschluss von Patienten mit Autoimmunerkrankungen } \\
\text { oder Immunsuppression }\end{array}$ & $\begin{array}{l}\text { - Diarrhö } \\
\text { - Hypertonus } \\
\text { - Appetitverlust } \\
\text { - Proteinurie } \\
\text { - Autoimmunreaktionen }\end{array}$ \\
\hline \multicolumn{3}{|l|}{ Folgelinie } \\
\hline $\begin{array}{l}\text { Regorafenib } \\
\text { Tablette, } 160 \text { mg täglich }\end{array}$ & $\begin{array}{l}\text { - Progress unter Sorafenib bei guter Verträglichkeit } \\
\text { - HCC im Stadium BCLCC } \\
\text { - Child-Pugh A } \\
\text { - ECOG } 0\end{array}$ & $\begin{array}{l}\text { - Hauttoxizität, v. a. HFSR } \\
\text { - Fatigue } \\
\text { - Diarrhö } \\
\text { - Transaminasenanstieg }\end{array}$ \\
\hline $\begin{array}{l}\text { Cabozantinib } \\
\text { Tablette, } 60 \text { mg täglich }\end{array}$ & $\begin{array}{l}\text { - Progress unter Sorafenib } \\
\text { - Unverträglichkeit von Sorafenib } \\
\text { - HCC im Stadium BCLCC } \\
\text { - Child-Pugh A } \\
\text { - ECOG } 0\end{array}$ & $\begin{array}{l}\text { - Hauttoxizität, v.a. HFSR } \\
\text { - Hypertonus } \\
\text { - Transaminasenanstieg } \\
\text { - Fatigue } \\
\text { - Diarrhö }\end{array}$ \\
\hline $\begin{array}{l}\text { Ramucirumab } \\
\text { Infusion, alle } 2 \text { Wochen } 8 \text { mg/kg KG }\end{array}$ & $\begin{array}{l}\text { - Progress unter Sorafenib } \\
\text { - Unverträglichkeit von Sorafenib } \\
\text { - HCC im Stadium BCLCC } \\
\text { - Child-Pugh A } \\
\text { - ECOG } 0 \\
\text { - AFP } \geq 400 \mathrm{ng} / \mathrm{ml}\end{array}$ & $\begin{array}{l}\text { - Hypertonus } \\
\text { - Hyponatriämie } \\
\text { - Proteinurie }\end{array}$ \\
\hline
\end{tabular}

Grundsätzlich sollte unter TKI-Therapie der Patient in regelmäßigen Abstand gesehen werden. Nach Einleitung der Therapie empfiehlt sich eine erste klinische Kontrolle nach 1-2 Wochen, dann im 4-Wochen-Intervall. Die Hauptnebenwirkungen beider TKI umfassen Durchfall, Hauttoxizität, v.a. Hand-Fuß-Haut-Reaktion (s. „Praxis Management der HFSR“), Fatigue, Hypertonus und Appetitverlust, weshalb ein konsequentes Nebenwirkungsmanagement essenziell ist.

\begin{abstract}
Merke
Aufgrund häufiger Nebenwirkungen sollen Patienten mit einer Tyrosinkinase-Inhibitoren-Therapie insbesondere bei Therapieeinleitung engmaschig gesehen werden.
\end{abstract}

Nach 2 negativen Phase-III-Monotherapie-Studien mit einem Immuncheckpoint-Inhibitor zeigte sich erstmalig in der Phase-III-Studie IMbrave150 ein signifikanter Nutzen einer Immuntherapie [15]. In dieser Studie wurde die Kombination von Atezolizumab, einem PD-L1-Inhibitor (PD-L1: programmed death-ligand 1), und Bevacizumab, einem VEGF-Inhibitor, im Vergleich zu Sorafenib als Erstlinientherapie untersucht. Das mediane Überleben 


\section{PRAXIS}

\section{Management der HFSR}

Am häufigsten betreffen kutane Nebenwirkungen der TKI die Handflächen und Fußsohlen (Hand-Fuß-Haut-Reaktion, HFSR oder palmar-plantare Erythrodysästhesie), wobei diese unter Lenvatinib deutlich seltener auftreten als unter Sorafenib, Regorafinib oder Cabozantinib [13]. Als hilfreich haben sich die folgenden Maßnahmen erwiesen: Entlastung von Druckstellen durch Tragen bequemer Schuhe, ggf. Pediküren und Maniküren und eine regelmäßige Haut-, Hand- und Fußpflege mit harnstoffhaltigen (5-10\%ig) Lotionen und Cremes. Bei den ersten Anzeichen einer HFSR sollten diese Maßnahmen intensiviert werden. Die Anwendung von glukokortikoidhaltigen Salben kann notwendig sein. Bei weiterer Verschlechterung der HFSR (Blasenbildung und/oder Ulzerationen) sollte die TKI-Dosierung reduziert oder die Therapie pausiert werden, um die komplette Heilung der Läsionen zu ermöglichen.

der Patienten, die mit Atezolizumab und Bevacizumab behandelt wurden, war zum Zeitpunkt der Interimsanalyse noch nicht erreicht. Die Patienten im Sorafenib-Arm hingegen überlebten im Median 13,2 Monate. Das progressionsfreie Überleben lag bei der Kombinationstherapie bei 6,8 Monaten, während es unter Sorafenib nur 4,3 Monate waren. Hierbei zeigten sogar 18 Patienten unter der Kombinationstherapie eine komplette und 71 eine partielle Remission, unter Sorafenib war lediglich bei 19 Patienten ein partielles Ansprechen nachweisbar. Diese Studie ist somit die bislang einzige positive Immuntherapie-Zulassungsstudie beim HCC. Die FDA-Zulassung (Food and Drug Administration) erfolgte am 29. Mai 2020, die EMA-Zulassung (Europäische ArzneimittelAgentur) wird im 3. Quartal 2020 erwartet.

Merke

Die Kombination aus Atezolizumab und Bevacizumab wird sich als neuer Standard in der Erstlinientherapie des HCC etablieren.

Aufgrund einer gering erhöhten Blutungsgefahr unter Bevacizumab müssen potenzielle Blutungsquellen, wie z. B. Ösophagusvarizen, vor Therapieeinleitung suffizient behandelt werden.

\section{Folgetherapien}

Aktuell können in der Folgetherapie des HCC 3 Medikamente nach einer Vortherapie mit Sorafenib bei Patienten mit erhaltener Leberfunktion (Child-Pugh A) eingesetzt werden. Aufgrund einer häufigen Verschlechterung der Leberfunktion im Rahmen der Erstlinientherapie kann nur ein Teil der Patienten mit HCC mit einer Folgetherapie behandelt werden.
Regorafenib ist ein primärer VEGFR-TKI, der im Vergleich zu Placebo nach Vortherapie mit Sorafenib eine Verbesserung des Gesamtüberlebens von 7,8 auf 10,6 Monate zeigte. Auch das progressionsfreie Überleben war mit 3,1 zu 1,5 Monaten doppelt so lang wie unter Placebo [16]. Die häufigsten schwerwiegenden Nebenwirkungen einer Behandlung mit Regorafenib waren Hypertonie, Fatigue, Diarrhöen und HFSR. Letztere sowie ein Transaminasenanstieg waren der häufigste Grund für einen Therapieabbruch. Wichtigstes Einschlusskriterium der Studie war eine Sorafenib-Verträglichkeit. Regorafenib steht derzeit in Deutschland nicht mehr zur Verfügung und muss über eine Auslandsapotheke importiert werden.

Ein weiterer Multi-TKI, Cabozantinib, wurde nach Progress oder bei Unverträglichkeit von Sorafenib in der Zweitlinie geprüft und zugelassen [17]. Hier zeigte sich ebenfalls eine deutliche Verbesserung des medianen Gesamtüberlebens (von 8,0 auf 10,2 Monate) sowie des progressionsfreien Überlebens (5,4 vs. 1,9 Monate). Schwerwiegende Nebenwirkungen umfassten in der Zulassungsstudie HFSR, Hypertonie, einen Transaminasenanstieg sowie Fatigue. Im Therapieverlauf sind häufig Dosisreduktionen erforderlich.

Ramucirumab ist als monoklonaler Antikörper gegen VEFGR2 die dritte zugelassene Substanz für die Zweitlinientherapie des HCC. Patienten mussten in der Zulassungsstudie einen AFP-Wert $\geq 400 \mathrm{ng} / \mathrm{ml}$ aufweisen und zuvor mit Sorafenib behandelt worden sein [18]. Ramucirumab zeigte ebenfalls einen deutlichen Überlebensvorteil gegenüber Placebo und weist im Vergleich zu den genannten TKI eine bessere Verträglichkeit auf. Die Zahl schwerwiegender Nebenwirkungen war geringer als unter einer TKI-Therapie und umfasste vor allem Hypertonie, eine Hyponatriämie sowie eine Proteinurie.

Merke

Patienten mit einem HCC im Stadium BCLC D sowie Patienten mit ECOG > 2 sollten supportiv und palliativmedizinisch behandelt werden.

\section{Substanzen in klinischer Entwicklung}

Eine Vielzahl an Immuntherapie-Kombinationen wird derzeit in der Erstlinientherapie des nicht resektablen HCC untersucht. Die nächsten Ergebnisse von zulassungsrelevanten Studien werden erwartet für

- die Kombination des PD-L1-Antikörpers Durvalumab mit dem CTLA-4-Antikörper (CTLA-4: cytotoxic T-lymphocyte-associated protein 4) Tremelimumab (HIMALAYA-Studie) sowie

- die Kombination des PD-1-Antikörpers (PD-1: programmed cell death protein 1) Pembrolizumab mit Lenvatinib (LEAP2-Studie). 


\section{FALLBEISPIEL}

\section{Staging und Therapie}

Bei unserer Patientin wird nun eine triphasische CT durchgeführt. Es zeigen sich ein ca. $3 \mathrm{~cm}$ großer, unscharf begrenzter, arteriell hypervaskularisierter zentraler Tumorknoten mit venösem Wash-out sowie mehrere Herde in beiden Leberlappen mit gleichem Kontrastverhalten ( $\vee$ Abb. 4 u. $>$ Abb. 5). Der größte dieser Herde liegt im linkslateralen Leberlappen und hat einen Durchmesser von ca. $3,5 \mathrm{~cm}$. Weder bildgebend noch laborchemisch gibt es Hinweise für eine Leberzirrhose. Somit wird die Diagnose eines multilokulären HCC mit Einbruch in den rechten Ductus hepaticus (BCLC-Stadium C, fortgeschritten) bei chronischer Hepatitis-B-Virus-Infektion gestellt. Endoskopisch werden eine portalhypertensive Gastropathie und Ösophagusvarizen ausgeschlossen.

Nach Beginn einer antiviralen Therapie mit Tenofovir 245 mg Tagesdosis wird eine systemische Therapie mit Atezolizumab (1200 mg i. v.) und Bevacizumab (15 mg/kg KG i. v.) im 3-wöchigen Intervall eingeleitet. Hierunter kommt es zu einem anhaltenden Tumoransprechen mit z. T. kompletter Devaskularisation der HCC-Knoten ( $>$ Abb. 4 u. $\triangleright$ Abb. 5). Die filiforme Stenose des Ductus hepaticus dexter bildet sich unter der Therapie komplett zurück, sodass der Gallengangsstent entfernt werden kann. Die initial bestehende Erhöhung der Cholestaseparameter sowie der Transaminasen ist ebenfalls komplett rückläufig. Bis dato wird die Therapie hervorragend vertragen und weiter fortgeführt.
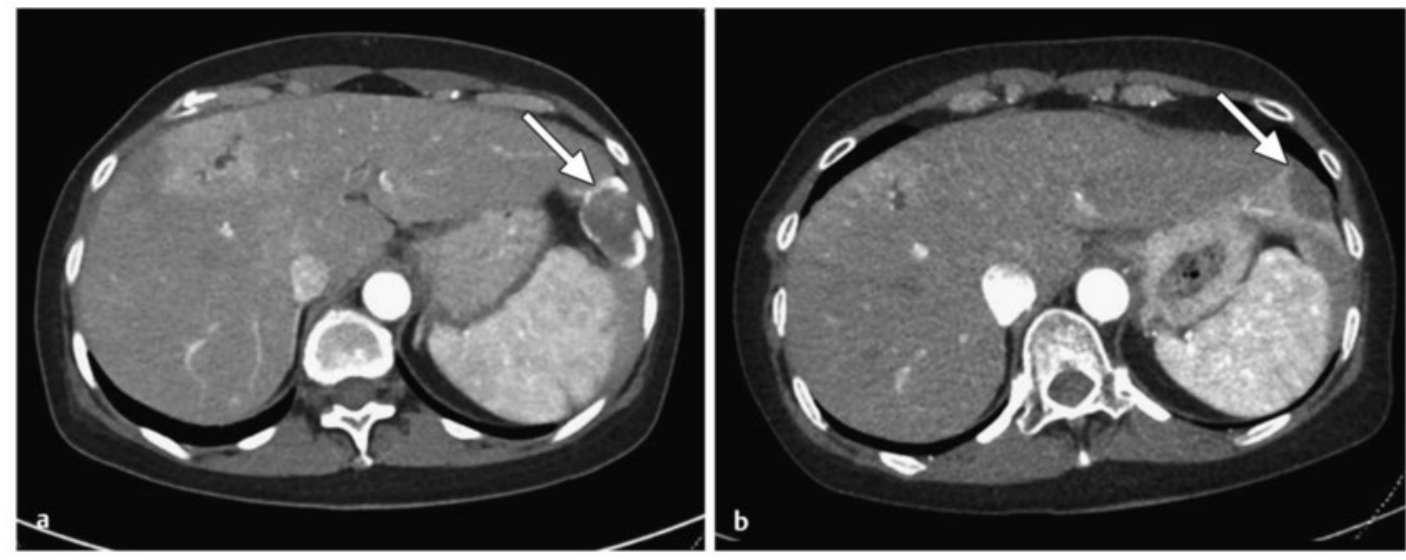

- Abb. 4 Triphasisches CT mit arterieller Darstellung eines peripheren Tumorknotens.

a Hypervaskularisierter Tumorknoten (Pfeil) vor Einleitung einer Therapie.

b Tumorknoten mit fehlender arterieller Kontrastierung unter Therapie mit Atezolizumab und Bevacizumab (Pfeil).
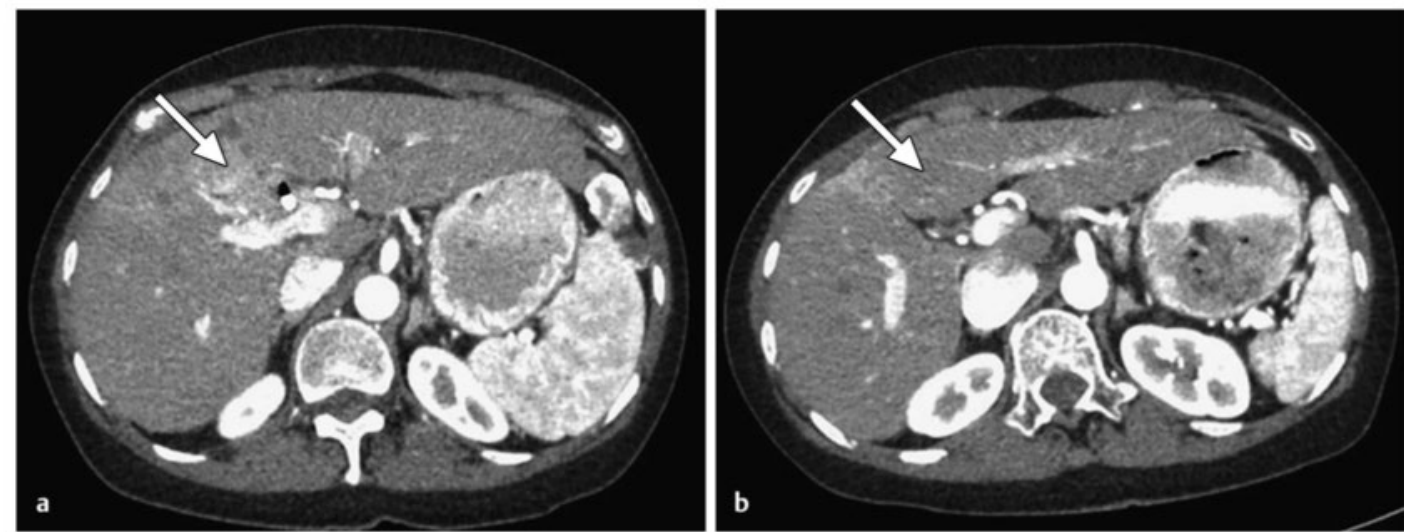

- Abb. 5 Triphasisches CT mit arterieller Darstellung eines zentralen Tumorknotens.

a Hypervaskularisierter Tumorknoten (Pfeil) vor Einleitung einer Therapie.

b Tumorknoten mit fehlender arterieller Kontrastierung unter Therapie mit Atezolizumab und Bevacizumab (Pfeil). 
Weitere derzeit rekrutierende Phase-III-Studien untersuchen u. a. den Nutzen

- der Kombination von Atezolizumab und Cabozantinib (COSMIC-Studie) bzw.

- der Kombination des PD-1-Antikörpers Nivolumab mit dem CTLA-4-Antikörper Ipilimumab (CheckMate 9DW-Studie) in der Erstlinientherapie.

Ein Schwerpunkt der klinischen Entwicklung der Immuntherapie beim HCC ist auch der Einsatz in früheren Tumorstadien. Mehrere Phase-III-Studien untersuchen den Nutzen von Immuncheckpoint-Blockern als Mono- oder Kombinationstherapie nach Resektion oder Ablation oder auch in Kombination mit TACE. Die aktuell vorliegenden Phase-II-Daten lassen einen erheblichen zusätzlichen Nutzen vermuten, der jedoch in randomisierten Studien zunächst bestätigt werden muss [19].

\section{KERNAUSSAGEN}

- Das hepatozelluläre Karzinom ist eine häufige Komplikation der Leberzirrhose und ist mit einer hohen Mortalität assoziiert.

- Die Zahl der Patienten, die ein HCC auf dem Boden einer NASH entwickeln, steigt zunehmend. Die sonografische Früherkennung soll allen Patienten mit Leberzirrhose angeboten werden.

- Die Schweregradeinteilung und Therapieentscheidung des HCC erfolgt anhand der BCLC-Klassifikation. Wesentlich hierfür sind das Ausbreitungsmuster des HCC und die Leberfunktion des Patienten.

- Die Therapie jedes Patienten mit HCC sollte interdisziplinär in einer spezialisierten Tumorkonferenz besprochen werden. Potenziell kurativ behandelbare Patienten mit HCC sollten operativ oder ablativ behandelt werden.

- Intraarterielle Verfahren stellen eine palliative Therapieoption dar, deren Einsatz immer kritisch geprüft werden muss.

- Zur systemischen Behandlung steht heute eine Vielzahl an gezielten Therapeutika für Patienten mit erhaltener Leberfunktion sowie in Kürze die erste Immuntherapie-Kombination zur Verfügung.
Interessenkonflikt

Erklärung zu finanziellen Interessen

Forschungsförderung erhalten: ja, von einer anderen Institution (Pharma- oder Medizintechnikfirma usw.); Honorar/geldwerten Vorteil für Referententätigkeit erhalten: ja, von einer anderen Institution (Pharma- oder Medizintechnikfirma usw.); Bezahlter Berater/interner Schulungsreferent/Gehaltsempfänger: ja, von einer anderen Institution (Pharma- oder Medizintechnikfirma usw.); Patent/Geschäftsanteile/Aktien (Autor/ Partner, Ehepartner, Kinder) an im Bereich der Medizin aktiven Firma: nein; Patent/Geschäftsanteile/Aktien (Autor/Partner, Ehepartner, Kinder) an zu Sponsoren dieser Fortbildung bzw. durch die Fortbildung in ihren Geschäftsinteressen berührten Firma: nein.

Erklärung zu nichtfinanziellen Interessen

Die Autorinnen/Autoren geben an, dass kein Interessenkonflikt besteht.

Autorinnen/Autoren

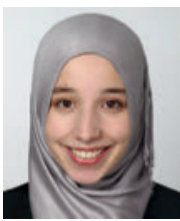

\section{Nada Abedin}

Dr. med., seit 2018 Weiterbildung zur Fachärztin für Innere Medizin und Gastroenterologie, Medizinische Klinik 1, Universitätsklinikum Frankfurt am Main.

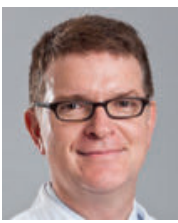

\section{Jörg Trojan}

Prof. Dr. med., Facharzt für Innere Medizin und Gastroenterologie. Leiter des Schwerpunkts Gastrointestinale Onkologie, Medizinische Klinik 1, Universitätsklinikum Frankfurt am Main.

Korrespondenzadresse

Prof. Dr. med. Jörg Trojan

Medizinische Klinik 1

Universitätsklinikum Frankfurt

Goethe-Universität Frankfurt

Theodor-Stern-Kai 7

60590 Frankfurt am Main

trojan@em.uni-frankfurt.de

Wissenschaftlich verantwortlich gemäß Zertifizierungsbestimmungen

Wissenschaftlich verantwortlich gemäß Zertifizierungsbestimmungen für diesen Beitrag ist Prof. Dr. med. Jörg Trojan, Frankfurt am Main. 
Literatur

[1] Galle PR, Former A, Llovet J et al. EASL Clinical Practice Guidelines: Management of hepatocellular carcinoma. J Hepatol 2018; 69: 182-236

[2] Globocan 2018. Cancer Fact Sheet: Liver Cancer. March 2019. Im Internet: http://gco.iarc.fr/today/data/factsheets/cancers/ 11-Liver-fact-sheet.pdf; Stand: 29.06.2020

[3] Kanwal F, Kramer JR, Mapakshi S et al. Risk of hepatocellular cancer in patients with non-alcoholic fatty liver disease. Gastroenterology 2018; 155: 1828-1837

[4] Kanwal F, Kramer JR, Asch SM et al. Long-term risk of hepatocellular carcinoma in HCV patients treated with direct acting antiviral agents. Hepatology 2020; 71: 44-55

[5] Vilar-Gomez E, Calzadilla-Bertot L, Wai-Sun Wong V et al. Type 2 diabetes and metformin use associate with outcomes of patients with non-alcoholic steatohepatitis-related, Child-Pugh A cirrhosis. Clin Gastroenterol Hepatol 2020. doi:10.1016/j. cgh.2020.04.083

[6] Vogel A, Cervantes A, Chau I et al. Hepatocellular carcinoma: ESMO Clinical Practice Guidelines for diagnosis, treatment and follow-up. Ann Oncol 2018; 29 (Suppl. 4): iv238-iv255

[7] Tzartzeva K, Obi J, Rich NE et al. Surveillance imaging and alpha fetoprotein for early detection of hepatocellular carcinoma in patients with cirrhosis: a meta-analysis. Gastroenterology 2018 ; $154: 1706-1718$

[8] Best J, Bechmann LP, Sowa JP et al. GALAD Score detects early hepatocellular carcinoma in an international cohort of patients with nonalcoholic steatohepatitis. Clin Gastroenterol Hepatol 2020; 18: 728-735

[9] Ioannou GN, Beste LA, Green PK et al. Increased risk for hepatocellular carcinoma persists up to 10 years after HCV eradication in patients with baseline cirrhosis or high FlB-4 scores. Gastroenterology 2019; 157: 1264-1278

[10] Mazzaferro V, Sposito C, Zhou J et al. Metroticket 2.0 Model for analysis of competing risks of death after liver transplantation for hepatocellular carcinoma. Gastroenterology 2018; 154: 128-139
[11] Bundesärztekammer. Richtlinien zur Organtransplantation gem. § 16 Abs. 1 S. 1 Nrn. 2 u. 5 TPG. Dtsch Arztebl 2013; 110: A-241/B-225/C-225

[12] Salem R, Lewandowski RJ, Mulcahy MF et al. Radioembolization for hepatocellular carcinoma using Yttrium-90 microspheres: a comprehensive report of long-term outcomes. Gastroenterology 2010; 138: 52-64

[13] Llovet JM, Ricci S, Mazzaferro V et al. Sorafenib in advanced hepatocellular carcinoma. N Engl J Med 2008; 359: 378-390

[14] Kudo M, Finn RS, Qin S et al. Lenvatinib versus sorafenib in first-line treatment of patients with unresectable hepatocellular carcinoma: a randomised phase 3 non-inferiority trial. Lancet 2018 ; 391: 1163-1173

[15] Finn RS, Qin S, Ikeda M et al. Atezolizumab plus bevacizumab in unresectable hepatocellular carcinoma. N Engl J Med 2020; 382: 1894-1905

[16] Bruix J, Qin S, Merle P et al. Regorafenib for patients with hepatocellular carcinoma who progressed on sorafenib treatment (RESORCE): a randomised, double-blind, placebo-controlled, phase 3 trial. Lancet 2017; 389: 56-66

[17] Abou-Alfa GK, Meyer T, Cheng AL et al. Cabozantinib in patients with advanced and progressing hepatocellular carcinoma. N Engl J Med 2018; 379: 54-63

[18] Zhu AX, Kang YK, Yen C] et al. Ramucirumab after sorafenib in patients with advanced hepatocellular carcinoma and increased $\alpha$-fetoprotein concentrations (REACH-2): a randomised, double-blind, placebo-controlled, phase 3 trial. Lancet Oncol 2019; 20: 282-296

[19] Brown Z], Greten TF, Heinrich B. Adjuvant treatment of hepatocellular carcinoma: prospect of immunotherapy. Hepatology 2019; 70: 1437-1442

Bibliografie

DOI https://doi.org/10.1055/a-1129-2608

Gastroenterologie up2date 2020; 16: 221-233

(c) Georg Thieme Verlag KG Stuttgart · New York ISSN 1616-9670 


\section{Punkte sammeln auf CME. thiemede}

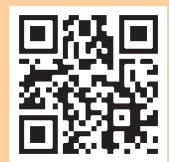

Diese Fortbildungseinheit ist in der Regel 12 Monate online für die Teilnahme verfügbar.

Den genauen Einsendeschluss finden Sie unter https://cme.thieme.de.

Sollten Sie Fragen zur Online-Teilnahme haben, finden Sie unter https://cme.thieme.de/hilfe

eine ausführliche Anleitung. Wir wünschen viel Erfolg beim Beantworten

der Fragen!

Unter https://eref.thieme.de/CXEQCQM oder über den QR-Code kommen Sie direkt zur Startseite des Wissenstests.

VNR 2760512020158721486

\section{Frage 1}

Welche Aussage zur Inzidenz des hepatozellulären Karzinoms (HCC) ist falsch?

A Die Inzidenz des HCC ist in den vergangenen Jahren gestiegen.

B Das HCC tritt meist auf dem Boden einer Leberzirrhose auf.

C Die Zahl der Patienten mit HCC ist weltweit gestiegen.

D Die Inzidenz des HCC ist in den vergangenen Jahren gesunken.

E Es sind mehr Männer als Frauen betroffen.

\section{Frage 2}

Welche Aussage zur Ätiologie des HCC ist richtig?

A Strahlenexposition trägt maßgeblich zur Entwicklung eines HCC bei.

B Alkoholkonsum spielt eine geringe Rolle bei der Entwicklung eines HCC.

C Die Zahl der Patienten mit HCC auf dem Boden einer HCV-Leberzirrhose steigt weiter signifikant an.

D Es kann nur eine Ätiologie für ein HCC vorliegen.

E Zunehmend werden HCC auf dem Boden einer nicht alkoholischen Steatohepatitis (NASH) diagnostiziert.

\section{Frage 3}

Was gehört nicht zu den etablierten Früherkennungs- und Präventionsmaßnahmen eines HCC?
A Alkoholkarenz
B halbjährliche Leberpunktion
$C$ halbjährliche Abdomensonografie
D Meidung hepatotoxischer Medikamente
E regelmäßige hepatologische Vorstellung

\section{Frage 4}

Welche Kombination diagnostischer Maßnahmen ist für das HCC nicht zu empfehlen?

A Ganzkörper-MRT + PET/CT (Positronenemissionstomografie/ Computertomografie)

B kontrastverstärkte Abdomensonografie + dynamische MRT

C dynamische MRT + Biopsie

D triphasische CT + kontrastverstärkte Abdomensonografie

E dynamische MRT + triphasische CT

\section{Frage 5}

Von wem sollten Therapieentscheidungen für HCC-Patienten getroffen werden?
A behandelnder Arzt
B Patient
C Klinikdirektor
D interdisziplinäre Tumorkonferenz
E interventioneller Radiologe

\section{Frage 6}

Bei welcher der folgenden Befundkonstellationen kommt die Lebertransplantation als kurative Therapie des HCC in Deutschland infrage?

A Child-Pugh-C-Leberzirrhose; 1 Herd von $7 \mathrm{~cm}$

B Child-Pugh-A-Leberzirrhose; 2 Herde, jeweils 2,1 cm

C Child-Pugh-B-Leberzirrhose; 4 Herde zwischen jeweils 2 und $3 \mathrm{~cm}$

D keine hepatische Vorerkrankung, 1 Herd von $6 \mathrm{~cm}$

E keine hepatische Vorerkrankung, 6 Herde zwischen 2 und $5 \mathrm{~cm}$

\section{Frage 7}

Welches der nachfolgend genannten Verfahren wird bei einem Patienten mit typischem singulärem HCC-Befund von 2 cm Größe im BCLC-Stadium A und Leberzirrhose Child-Pugh A empfohlen?
A selektive interne Radiotherapie (SIRT)
B Thermoablation
C transarterielle Chemoembolisation (TACE)
D Verlaufskontrolle
E Systemtherapie

\section{- Weitere Fragen auf der folgenden Seite...}




\section{Punkte sammeln auf CMIE.thieme.de}

Fortsetzung $\ldots$

\section{Frage 8}

Welche schwerwiegende Nebenwirkung führt bei Patienten unter systemischer Therapie mit Sorafenib oder Cabozantinib häufig zum Therapieabbruch?
A Proteinurie
B Obstipation
C Hypotonie
D Gewichtszunahme
E Hand-Fuß-Haut-Reaktion

\section{Frage 10}

Welche Behandlung kann auch bei Unverträglichkeit gegenüber Sorafenib in der Zweitlinie zur Anwendung kommen?
A Atezolizumab/Bevacizumab
B Lenvatinib
C Cabozantinib oder Ramucirumab
D Regorafenib
E Es ist keine Therapie möglich.

\section{Frage 9}

Zu welcher Kombinationstherapie liegen zulassungsrelevante Daten für die Erstlinientherapie des HCC vor?
A Pembrolizumab/Lenvatinib
B Atezolizumab/Bevacizumab
C Nivolumab/Ipilimumab
D Atezolizumab/Cabozantinib
E Durvalumab/Tremelimumab

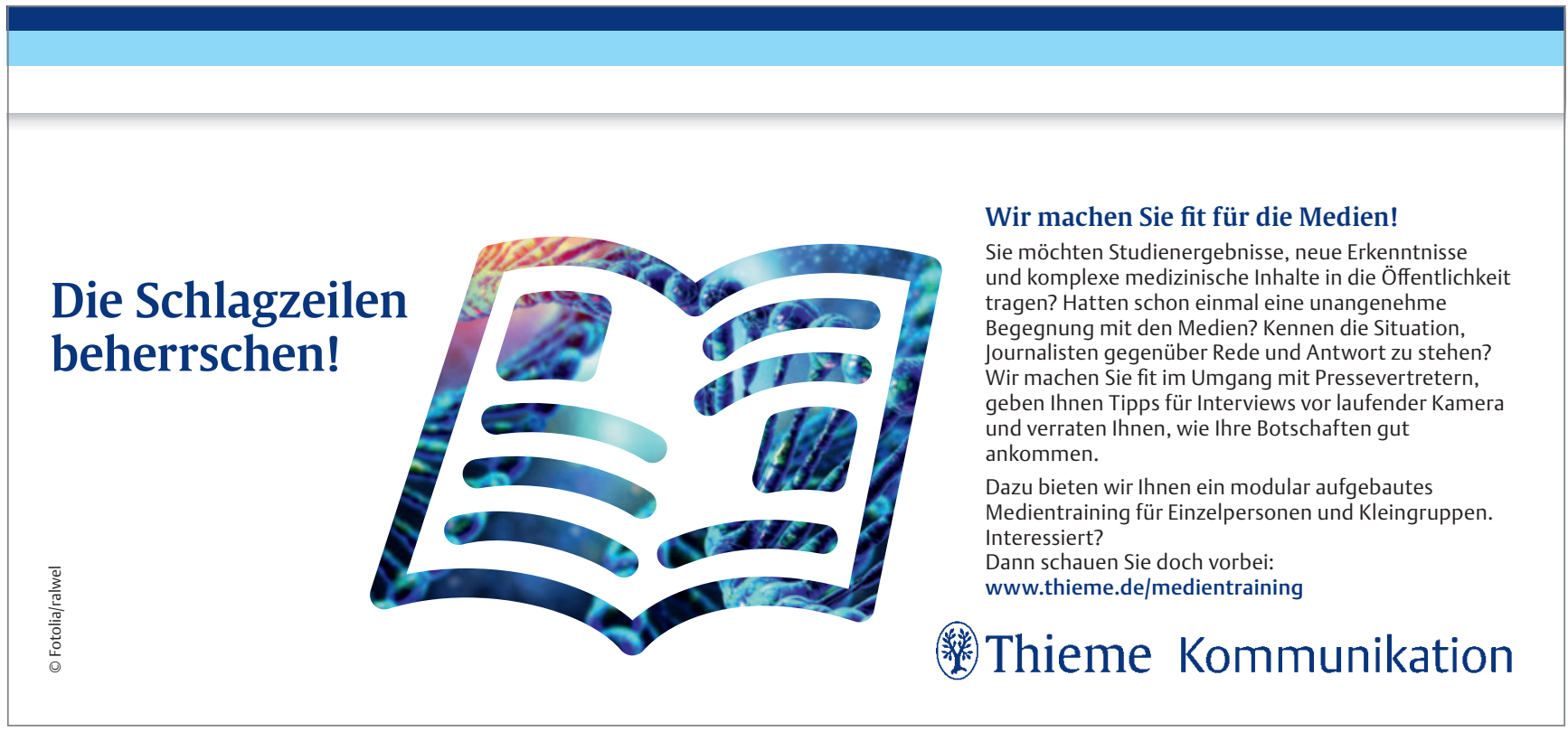

